\title{
Longitudinal Analysis of Sleep-Wake States in Neonatal Rats Subjected to Hypoxia-Ischemia
}

\author{
Xiaowei Sun', Fenqin Xue², Jialin Wen', Limin Gao', Yang Li', Lijun Yang', Hong Cui' \\ 'Department of Pediatrics, Beijing Friendship Hospital, Capital Medical University, Beijing, People's Republic of China; ${ }^{2}$ Department of Core Facility \\ Center, Capital Medical University, Beijing, People's Republic of China
}

Correspondence: Lijun Yang; Hong Cui, Beijing Friendship Hospital, Capital Medical University, No. 95 Yongan Road, Xicheng District, Beijing, I00050 People's Republic of China, Email yanglijun1979@ccmu.edu.cn; cuihong100@ccmu.edu.cn

Objective: Sleep is necessary for brain maturation in infants. Perinatal hypoxic-ischemic encephalopathy (HIE) is a major cause of chronic neurological disease in infants. Although the developmental changes of electroencephalogram (EEG) in human newborns have been described, little is known about the EEG normal maturation characteristics in rodents and the changes in sleep-awake states caused by hypoxia-ischemia (HI). This study aimed to investigate the pathological response of sleep-wake states in neonatal rats with HIE.

Methods: We constructed HIE and sham models on postnatal day (P) 3 rats and continuously monitored them using electroencephalography and electromyography for up to P12. The distribution of sleep-wake states was analyzed to estimate the effects of HIE. Results: Compared with the sham group, the HI group showed lower rapid eye movement (REM) sleep percentage, but wake percentage and frequency was higher during P4-P12. The frequency of REM and non-rapid eye movement (NREM) sleep increased and the duration of REM and NREM sleep decreased after HI induction. However, it gradually returned to the normal level with an increase in daytime.

Conclusion: HI damage alters the sleep-wake patterns during early neural development. The findings provide a comprehensive assessment of serial sleep-wake state recordings in neonatal rats from P4-P12.

Keywords: electroencephalography, EEG, hypoxia-ischemia, rapid eye movement, premature

\section{Introduction}

Hypoxic-ischemic encephalopathy (HIE) is one of the main causes of brain injury in preterm infants. ${ }^{1,2}$ The incidence of HIE is higher in preterm infants (4-48 per 1000 preterm newborns) than in term infants (1-8 per 1000 live births), indicating that hypoxia-ischemia (HI) is a primary contributor to pathophysiology of preterm brain injury. ${ }^{3-5}$ Focal or diffuse brain damage caused by HI has significant effects on the network of the sleep-wake cycle and EEG changes. ${ }^{6}$ The maintenance of the sleep-wake cycle involves multiple regions of the brain and reflects the maturation, integrity, and stability of the neuronal circuit. ${ }^{7,8}$

The sleep-wake cycle includes wakefulness, rapid eye movement (REM) sleep, and non-rapid eye movement (NREM) sleep, which is the physiological representations of the intricate interactions of brain structures, including the basal forebrain, thalamic, hypothalamus. ${ }^{7,9}$ Electroencephalography (EEG) and electromyography (EMG) are excellent assessment modalities of the sleep-wake cycle. ${ }^{9}$ EEG and amplitude-integrated EEG (aEEG) findings have good prognostic value for neurodevelopmental outcomes in neonatal hypoxic-ischemic encephalopathy (HIE). ${ }^{10-12}$ However, although the developmental changes of EEG in human newborns and the abnormalities of EEG caused by HIE have been characterized in detail, and there are few studies on normal maturational features of rodent EEGs, ${ }^{13,14}$ the changes in sleep-awake vigilance states caused by HI remain poorly understood.

Animal models are crucial for investigating the pathological and physiological mechanisms involved in neurological disorders affecting the human neonates and infants. However, few studies of EEG and EMG studies have been conducted in neonatal rats with HIE because of some technical limitations (eg, small head size, an unfixed skull, and the need for neonatal rats to live with their mothers for breastfeeding support) and other factors. As rats represent the most commonly 
used species for translational research, the use of continuous recording techniques in rats to comprehensively assess EEG characteristics and the sleep-wake cycle during neonatal development would be of great value in the study of normal brain maturation and HIE during the acute stage. At postnatal day 3 (P3), rats are similar to very preterm infants with respect to the stage of oligodendroglial maturation and axonal outgrowth, making them highly suitable for use in such studies. ${ }^{15,16}$ Therefore, the objective of this study was to investigate the pathological response of sleep-wake states in neonatal rats with HIE. Towards this goal, we assessed the EEG characteristics and vigilance state changes from postnatal days 4 to 12 in a rat model of HIE and sham group by using serial EEG and EMG recordings.

\section{Materials and Methods}

\section{Animals}

All Sprague-Dawley rats used in this study were obtained from Beijing Vital River Laboratory Animal Technology Co., Ltd. and were reared and housed under standardized conditions $\left(22 \pm 1{ }^{\circ} \mathrm{C}, 55 \%\right.$ relative humidity, 12 -h light-dark cycle, lights on at 8:00) with free access to water and food ad libitum. All animal experiments were carried out after approval by the Local ethics committee (Beijing Friendship Hospital Ethics Committee, Capital Medical University) and in compliance with the "Laboratory animals-General requirements for animal experiment" (GB/T 35823-2018, China).

\section{Electrode Implantation}

P2 rats (mean weight: $7.78 \mathrm{~g} ; \mathrm{n}=20$ ) were surgically implanted with two EEG electrodes, two EMG electrodes, and one reference electrode. Anesthesia was induced with $2 \%$ isoflurane and maintained with $1.5 \%$ isoflurane and $30 \%$ oxygen. After a midline vertical incision to expose the skull, forceps were used to remove any connective tissue, and the skull was cleaned and dried with $3 \%$ hydrogen peroxide for electrode placement. Two holes $(0.5 \mathrm{~mm})$ for EEG electrode implantation were made $2 \mathrm{~mm}$ before the lambda and $2 \mathrm{~mm}$ lateral from midline sutures using a hand-held microdrill. In addition, a $1 \mathrm{~mm}$ hole was drilled $3 \mathrm{~mm}$ under the lambda to allow implantation of the reference electrode. Two tungsten electrodes (0.003-inch outer diameter; A-M Systems, 131 Business Park Loop Sequim, WA, USA) were implanted bilaterally in the parietal cortex with a small amount of dental cement added to secure the electrodes to the skull. Two insulated stainless-steel wire electrodes were inserted into the nuchal muscles for EMG recordings, with the wire bent to follow the curvature of the head and neck. The five electrodes were connected to a 6-pin electrode pedestal, and the weight of the entire apparatus was approximately $220-230 \mathrm{mg}$. After the procedure, the pups were allowed to recover until the return of spontaneous movements, following which they were reunited with their mothers.

\section{Neonatal Rat Model of Hypoxic-Ischemic Brain Injury}

P3 neonatal rats $(n=20)$ were allocated to the experimental $(n=10)$ and sham control groups $(n=10)$ using the random Excel function. After induction of anesthesia with isoflurane, the neck area was prepared and draped in a sterile manner. Next, a small midline incision was made on the anterior neck, and the left carotid artery was isolated and double-ligated with a surgical suture. The artery was severed between the ligations. All surgeries lasted for no longer than 10 minutes. After completion of the surgical procedure, the pups housed in a warm cage $\left(38{ }^{\circ} \mathrm{C}\right)$ until the return of spontaneous movements, and then returned to their mothers for a total recovery time of $120 \mathrm{~min}$. Thereafter, they were then placed in an airtight jar with $8 \%$ oxygen and balanced with $92 \%$ nitrogen for $2 \mathrm{~h}$ at $37^{\circ} \mathrm{C}$. In the sham control group, the left carotid artery was isolated without ligation or hypoxic treatment.

\section{Data Acquisition and Recording}

Neonatal rats were separated from the dam and underwent video-EEG and EMG monitoring between P4 and P12. All pups in the HI and sham groups were separated from their mothers and underwent an hour of EEG and EMG monitoring at the same time every morning between $\mathrm{P} 4$ and P12. After recording, the pups were immediately returned to their mothers. The detailed workflow is shown in (Figure 1). Each monitored neonatal rat was provided a special self-made nest and the signals from multiple receivers were collected at a sampling rate of $1000 \mathrm{~Hz}$ and stored on a PC hard disk (Figure 2). EEG signals were 


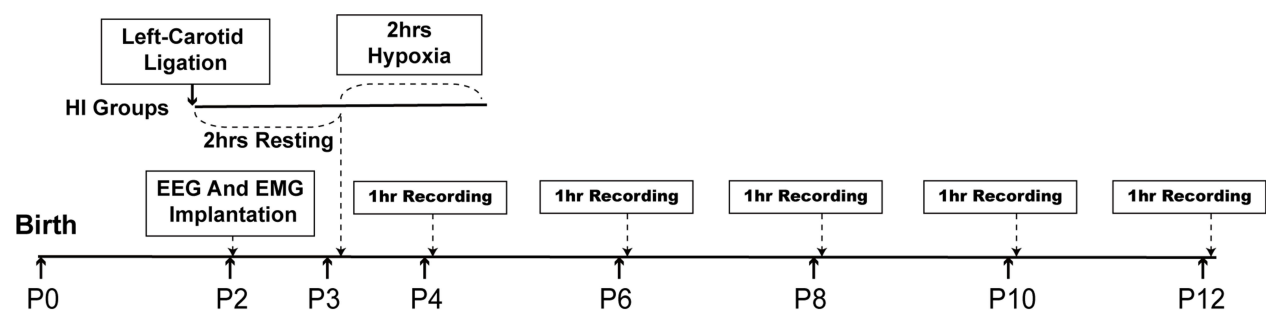

Age (Postnatal days)

Figure I Schematic illustration of the time course of the experimental design in HI group rats. P, postnatal day, hrs, hours.

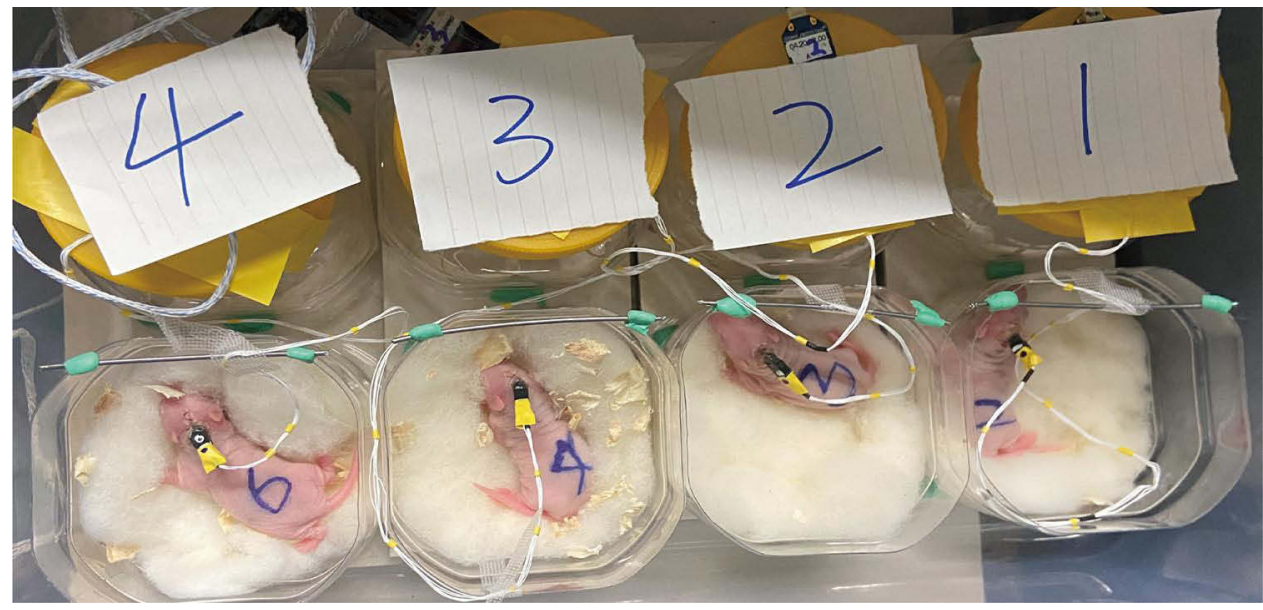

Figure 2 Neonatal rats in sleep monitoring.

filtered with a band-pass between $0.5 \mathrm{~Hz}$ and $40 \mathrm{~Hz}$. High-pass and low-pass filters were set at $0.5 \mathrm{~Hz}$ and $80 \mathrm{~Hz}$ for EMG signals, respectively.

\section{Statistical Analysis}

NeuroExplorer 5.0 (Nex Technologies) was used to open the stored data file and analyze the EEG and EMG data. Student's $t$-test was used to compare the HI and sham group rats. Quantitative differences between ages were analyzed by one-way repeated measures ANOVA followed by Tukey's test. Statistical significance was defined as $\mathrm{P}<0.05$.

\section{Results}

\section{Identification of Vigilance States with EEG and EMG Characterization of the Developing Neonatal Rats}

EEG and EMG were used to identify vigilance states during the development of neonatal rats from P4 to P12. In P4 rats (Figure 3A), The features of REM sleep were characteristic of continuous low-amplitude and mixed-frequency EEG with unobvious EMG activity. NREM sleep was characterized by discontinuous relatively higher amplitude activity with low nuchal muscle tone. The awake state was characterized by discontinuous traces of low amplitude EEG related to active EMG. By P6 (Figure 3B), the EEG and nuchal EMG displayed a low amplitude continuous EEG activity with inhibited EMG during REM sleep. There were discontinuous relatively high amplitude bursts of slow wave EEG activity with suppressed nuchal muscle tone among NREM. In addition, we noted relatively continuous low amplitude EEG activity and relatively obvious EMG traces during wakefulness. In P8 rats (Figure 3C), further development of the EEG patterns made the discrimination among REM sleep, NREM sleep, and wake states. The NREM sleep period demonstrates a more 
A

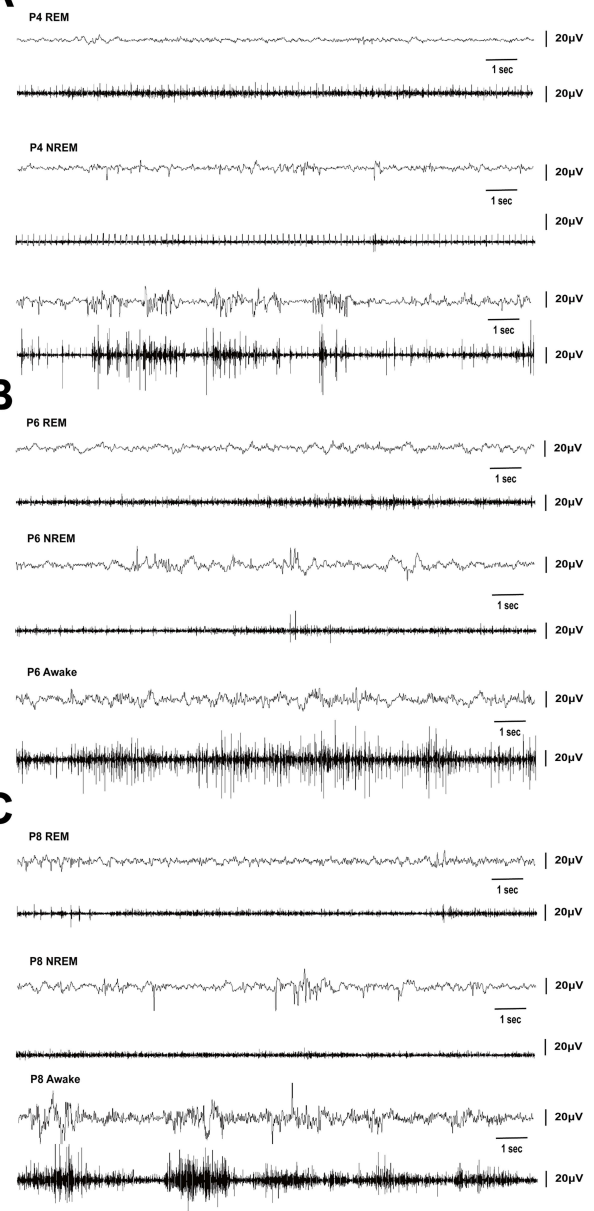

D
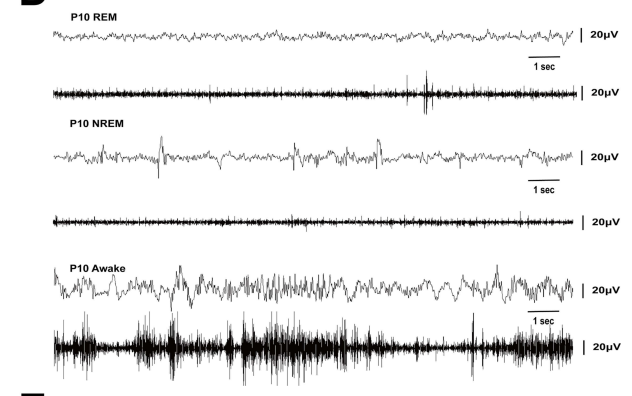

$\mathbf{E}$

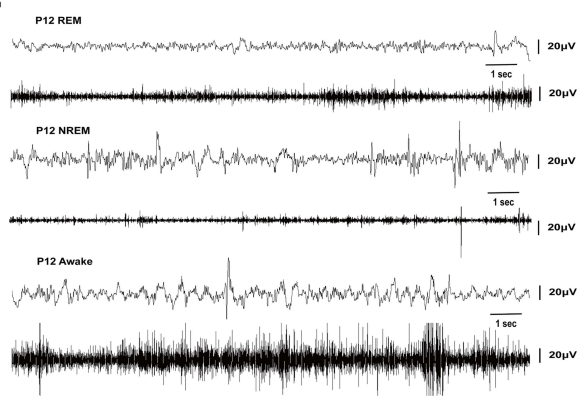

Figure 3 Identification of vigilance states in the neonatal rats from P4 to PI2. (A) Representative EEG and EMG activities of a neonatal rat on postnatal day 4 (P4) show a discontinuous EEG activity pattern during periods of muscle atonia (upper traces) and high muscle tone (lower traces). (B) Representative P6 EEG and nuchal EMG display a low-amplitude EEG activity with inhibited EMG during REM sleep, and relatively high-amplitude discontinuous traces of prominent slow wave activity on EEG and decrease EMG traces during NREM, and low amplitude with relatively continuous EEG activity and obvious EMG activity among the awake state. (C) Representative P8 EEG, EMG patterns display well-defined distinction among REM, NREM, and awake patterns. (D) Representative EEG, EMG traces obtained on PI0 display a low amplitude continuous EEG activity with oppressed EMG during REM sleep, and higher amplitude discontinuous activity on EEG during NREM sleep, and high amplitude continuous EEG traces and obvious EMG traces during the awake period. (E) Representative PI2 EEG and EMG traces display clear vigilance state patterns. V, volts, S, seconds, P, postnatal day. 
continuous and higher amplitude EEG than P4. In P10 rats (Figure 3D), EEG and EMG traces displayed continuous EEG activity with or without short myoclonic jerks during REM sleep and relatively higher amplitude discontinuous bursts on EEG during NREM sleep. The awake state was characterized by serial EEG activity and obvious EMG activity. By P12 (Figure 3E), EEG and EMG traces displayed clear vigilance state patterns. REM and NREM sleep demonstrated increased amplitude continuous bursts on EEG. In general, the EEG patterns evolved from an unobvious low-amplitude discontinuous pattern into an increasingly distinct vigilance state.

\section{Developmental Changes in the Percentage of Time of Vigilance States in Neonatal Rats of the Sham Groups and HI Groups}

In the sham group, the percentage of REM sleep was the highest in P4 rats. However, the percentage of REM sleep decreased gradually with an increase in the number of days of birth (Figure 4A). Meanwhile, NREM sleep showed the opposite trend. The proportion of NREM sleep was the lowest at P4, and NREM sleep gradually became the main form of sleep state with the development of the pups (Figure 4B). The proportion of wakefulness was low during P4-P10, but increased at P12, and the difference was statistically significant (Figure 4C). This may indicate that neonatal rats spend a significant proportion of their recorded sleep time before P10, while the proportion of awake increased gradually after P10.

Compared with the sham group, the HI group demonstrated lower percentages in REM sleep percentages during P4P12, especially during P4-P8 (Figure 4A). The proportion of NREM sleep increased in P4, but decreased in P10 group (Figure 4B). The proportion of awake time in neonatal rats increased significantly at P4, P6, P10 and P12 (Figure 4C). In summary, the overall trend in the HI group was closer to that in the sham group with respect to the percentage of REM, NREM, and WAKE with increasing day age.

\section{Developmental Changes in the Frequencies of Vigilance States in Neonatal Rats in the Sham and HI Groups}

In the sham group, the serial frequencies of REM sleep and NREM sleep did not change significantly through P4-P12 during $1 \mathrm{~h}$ of sleep (Figure 5A and B). In the HI group, the frequency of REM sleep decreased gradually with the increase in day age. The NREM was lower in P12 than in P4, and the frequency of wake did not change significantly during P4-P12.

Compared with the sham group, the HI group showed significantly higher occurrences of REM sleep and NREM sleep in one hour during P4-P8, but there was no obvious difference in P10 and P12 (Figure 5A and B). Awakening frequency was also higher than in the sham group between P4 and P12 (Figure 5C). In summary, the frequency of REM and NREM events increased within 5 days after HI induction. However, it gradually returned to the normal level with an increase in daytime, and the neonatal rats in the HI group showed frequent awakening for a long time after HI induction.

\section{Developmental Changes in the Bout Duration of Vigilance States and Sleep-Wake (S- W) Cycles in Neonatal Rats of the Sham Group and HI Groups}

Bout lengths of REM sleep in the sham and HI groups did not change significantly with an increase in day age (Figure 6A). However, the duration of REM sleep between P4 and P8 in the HI group was lower than that in the sham group (Figure 6A). Compared with the sham group, the HI group showed higher lengths of NREM sleep at P12 and lower at P6-P10 (Figure 6B). There was no significant change in the lengths of the awake between P4 and P10 in the sham and HI groups, but the duration of wakefulness at P12 was higher than that at younger age. The bout lengths of awake at P6 and P8 in the HI group were shorter than those in the sham group (Figure 6C). In summary, HI induction contributed to a decline in the bout length in neonatal rats, but bout length gradually improved after P10.

There was no significant change in the duration of the sleep-wake cycles between P4 and P12 in the sham group. The lengths of the sleep-wake cycle were similar during P4-P12 in the HI group. Compared with the sham group, the HI group had relatively shorter lengths of the sleep-wake cycles at P8 and P10 (Figure 7). 
A

A

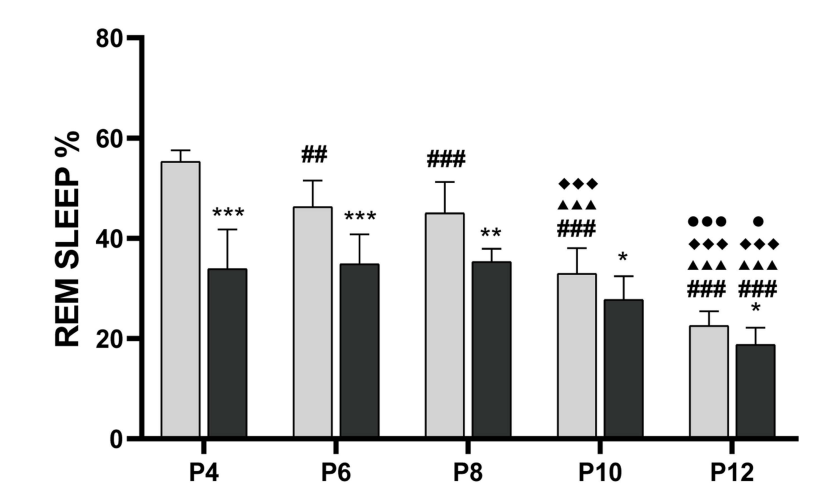

B

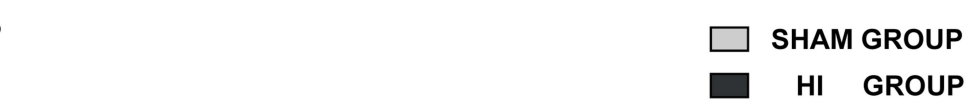

SHAM GROUP

HI GROUP

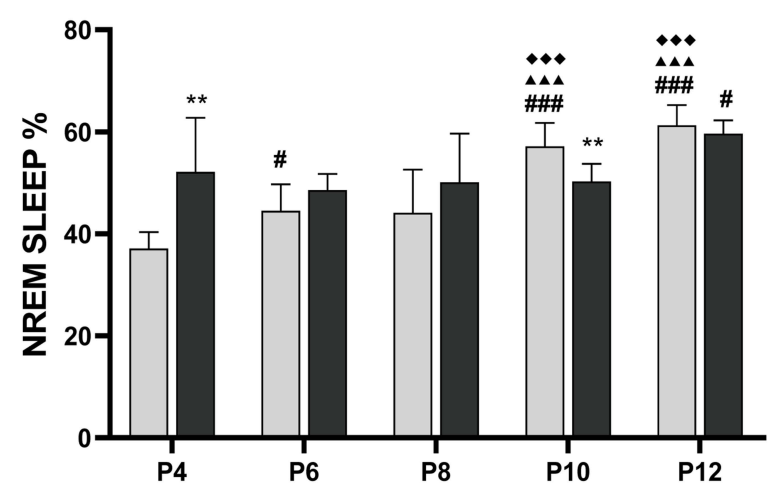

C

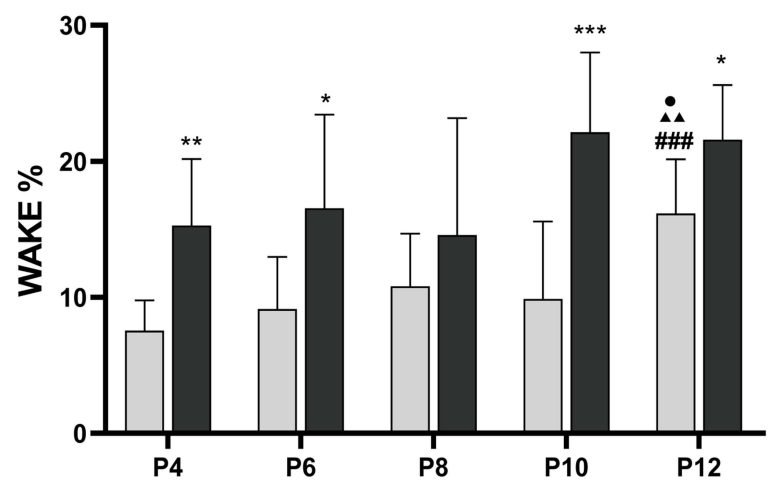

Figure 4 Developmental changes of the percentage of time in REM sleep (A), NREM (B), and wake (C) state on different postnatal days in the sham group and HI group. The $y$-axis represents the percentage of time spent in REM sleep $(\mathbf{A})$, NREM (B), and wake $(\mathbf{C})$ per hour on different postnatal days ( $x$-axis) in the sham and HI group neonatal rats. The comparison between the $\mathrm{HI}$ group and the sham group is depicted in *; the comparison of P4 values with other postnatal days in each group is shown in \#; the comparison of P8 values with PIO and PI 2 in each group is shown in $\bullet$; and the comparison of PI0 values with PI2 values in each group is shown in $\bullet$. *,\#, $\bullet$ P<0.05; **, \#, $\mathbf{\Delta} \boldsymbol{\Delta}, \mathrm{P}<0.0 \mathrm{I}$; ***, \#\#, $\boldsymbol{\Delta} \mathbf{\Delta} \boldsymbol{\Delta}, \diamond \diamond, \bullet \bullet \bullet P<0.001$. 
A

\section{SHAM GROUP}

HI GROUP

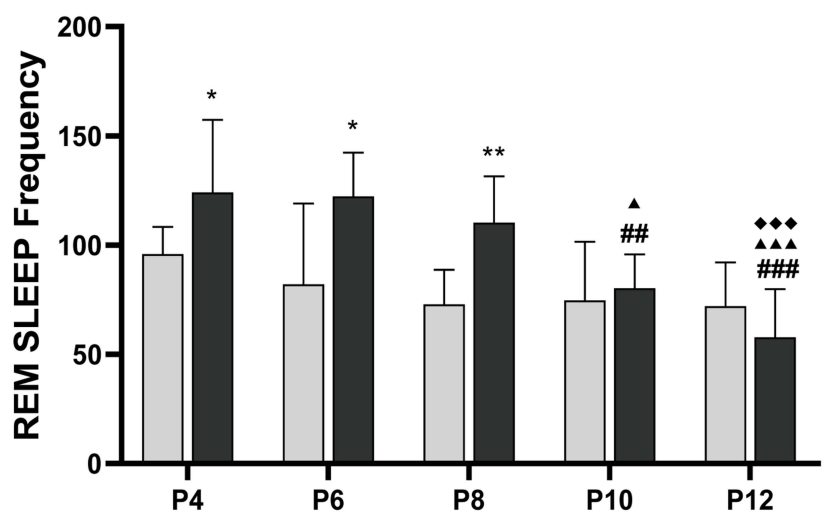

B

SHAM GROUP

HI GROUP

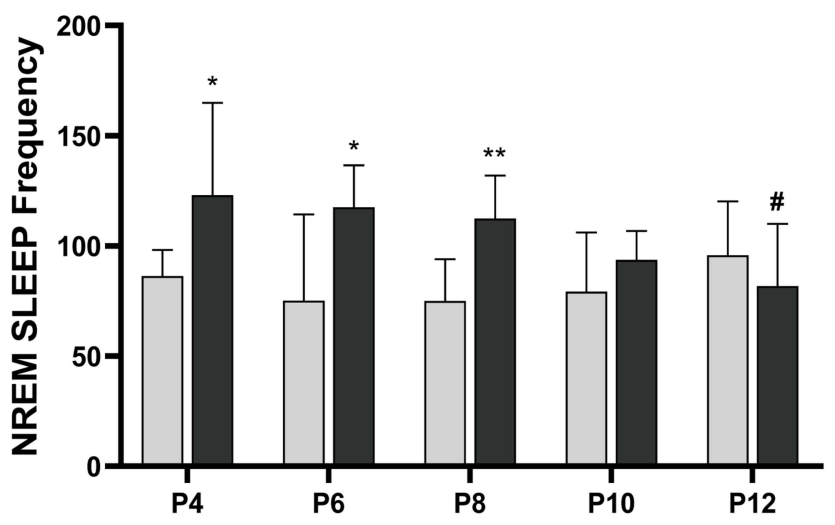

C

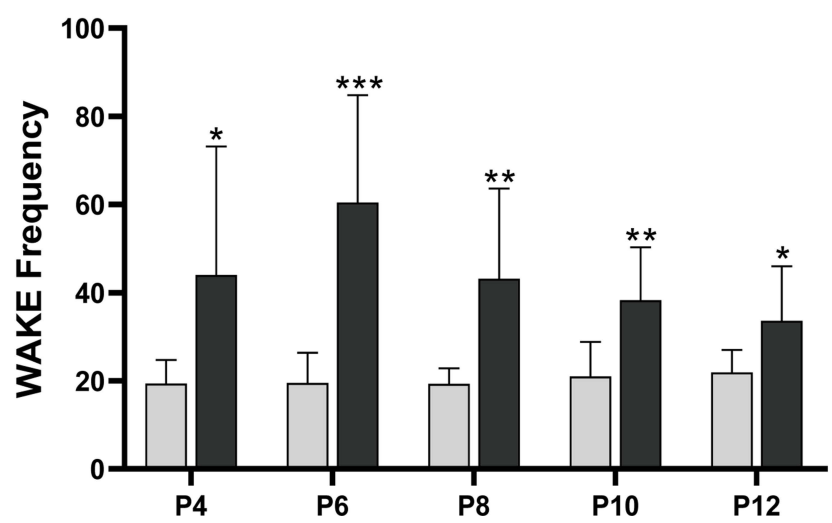

Figure 5 Number of the episodes of REM sleep (A), NREM sleep (B) and wake (C) on different postnatal days in the sham group and HI group. The $y$-axis shows frequencies of time spent during REM sleep (A), NREM (B), and wake (C) in an hour on different postnatal days ( $x$-axis) in sham and HI group neonatal rats. The comparison between the $\mathrm{Hl}$ group and the sham group is depicted in *; the comparison of P4 values with other postnatal days in each group is shown in \#; the comparison of P6 values with P8, PIO and PI2 in each group is shown in $\mathbf{\Delta}$; the comparison of P8 values with PI0 and PI2 in each group is shown in $\bullet$. $, \#, \mathbf{\Lambda}, \mathrm{P}<0.05 ; * *, \ldots \#, \mathrm{P}<0.01$; ***, \#\#, $\Delta \Delta \Delta, \diamond \diamond \bullet, P<0.001$. 
A

SHAM GROUP

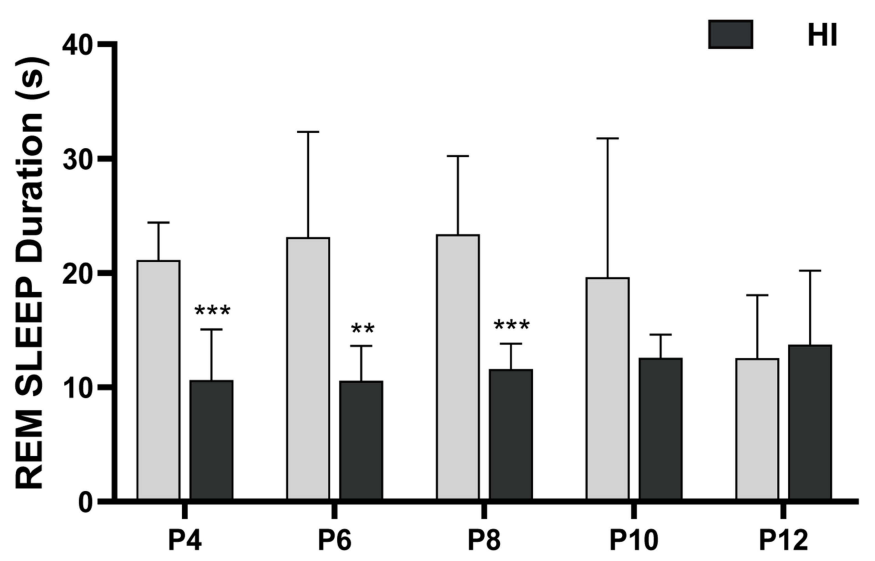

B

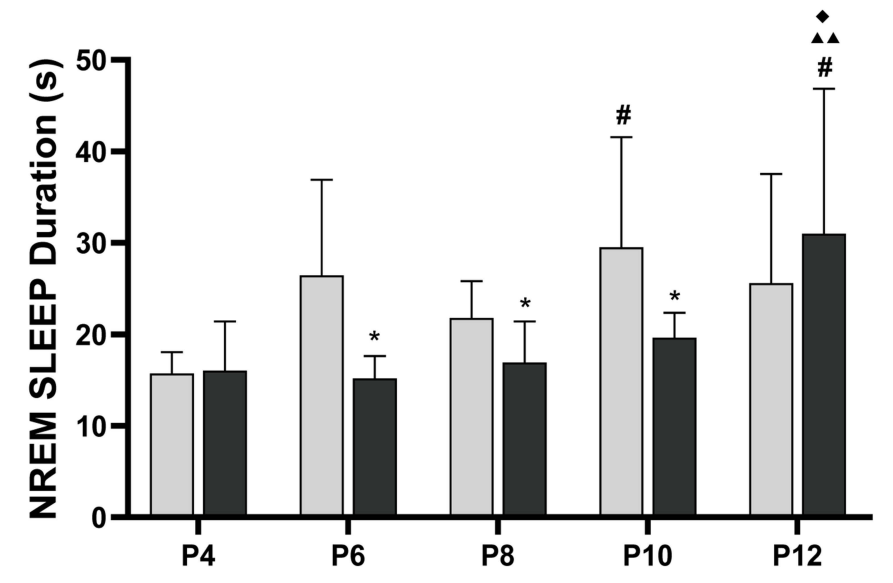

C

\section{SHAM GROUP}

HI GROUP

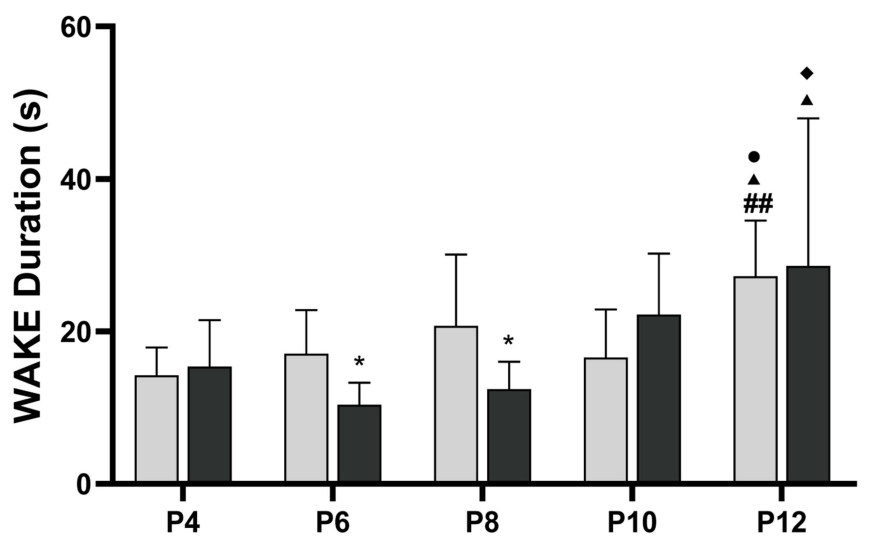

Figure 6 Bout duration of REM sleep (A), NREM sleep (B), and wake (C) in neonatal rats on different postnatal days in the sham group and HI group. The $y$-axis demonstrates the bout duration in REM sleep (A), NREM (B), wake (C) on different postnatal days ( $x$-axis). The comparison between the $H$ group and the sham group is depicted in *; the comparison of P4 values with other postnatal days in each group is shown in \#; the comparison of P6 values with P8, PI0 and PI2 in each group is shown in $\mathbf{\Lambda}$; the comparison of P8 values with PI0 and PI2 in each group is shown in $\bullet$, the comparison of PI0 values with PI2 in each group is shown in $\bullet *, \#, \mathbf{\Lambda}, \bullet, \bullet P<0.05 ; * *$, \#, $\Delta \Delta \mathrm{P}<0.01 ; * * * \mathrm{P}<0.01$. 


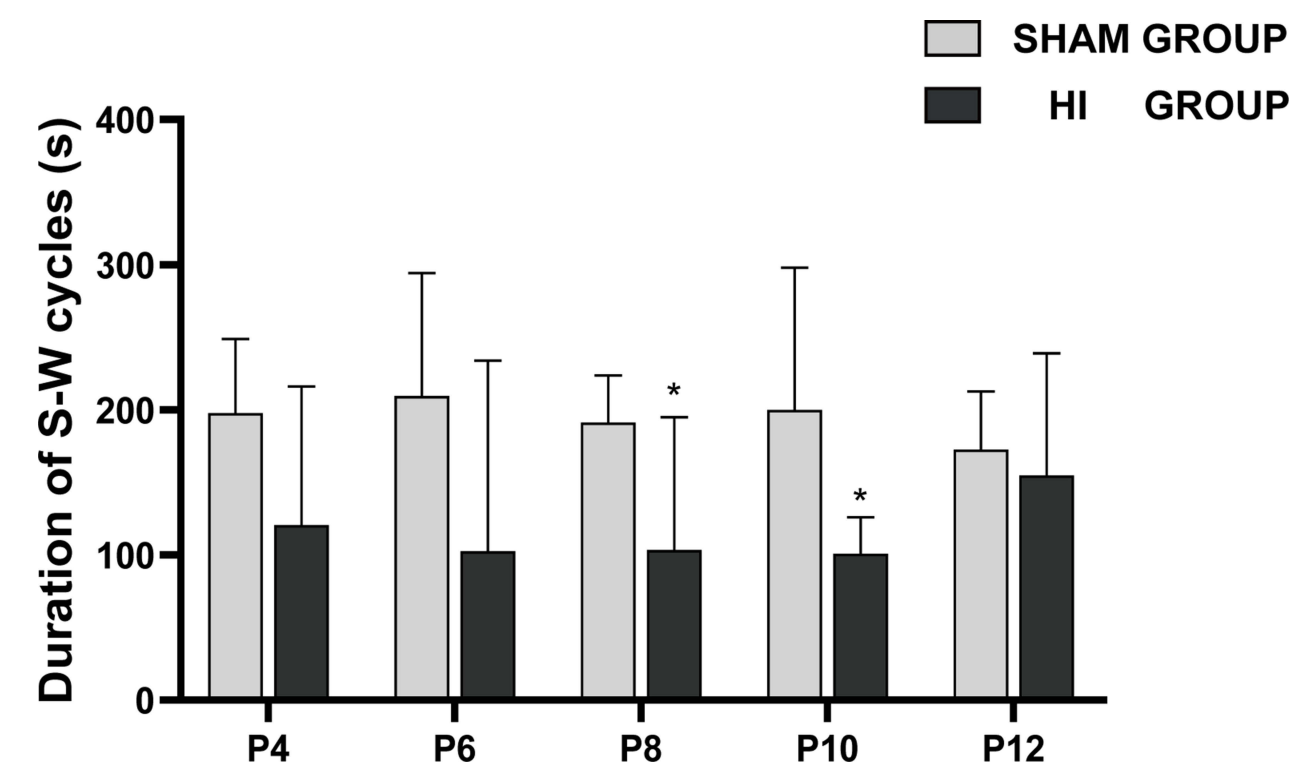

Figure 7 Changes in duration of sleep-wake $(\mathrm{S}-\mathrm{W})$ cycles on different postnatal days in the sham group and HI group. The $y$-axis represents the duration of S-W cycles on different postnatal days (x-axis) in sham and $\mathrm{HI}$ group neonatal rats. The comparison between the $\mathrm{HI}$ group and the sham group is depicted in $*$, $* \mathrm{P}<0.05$.

\section{Discussion}

The percentage of REM sleep at P4 was high in neonatal rats in the sham group, but it decreased gradually with an increase in day age. The rats followed a pattern of with increased NREM sleep and corresponding decreased REM sleep during the neonatal periods. This sleep pattern was quite similar to that observed in premature infants and neonates. ${ }^{17}$ To our knowledge, this is the first study to perform continuous EEG and EMG monitoring in a rat model of HIE at P3 to study sleep and wake states.

However, the REM sleep percentages in the rats from HI group were lower during P4-P12 and the wake time was higher than in the sham group, suggesting that HI affected the normal sleep-wake structure. In the acute phase of HIE, human neonatal sleep shows a decrease in the proportion of REM and an increase in the proportion of NREM and wake, ${ }^{6}$ and similar results were found in our HIE model of neonatal rats.

In our study, the duration of REM sleep between P4 and P8 was lower in the HI group than that in the sham group, but the NREM bout lengths remained unchanged at P4. In infants, REM sleep bout lengths are shorter and NREM sleep bout lengths remain unchanged in the asphyxia group during the first 3 days after birth. ${ }^{6}$ In neonatal animal models, the development of neural activity sensorimotor depends more on REM sleep compared with wakefulness. ${ }^{18-20}$ Consequently, changes (sleep deprivation or suppression) in sleep patterns can impair synaptic plasticity and cortical activity levels. ${ }^{21,22}$ In human infants, somatotopic cortical activity and learning are associated with sleep activity induction. ${ }^{23,24}$ In human adults, the locus coeruleus activity plays a key role in promoting wakefulness, while inhibition of its activity fosters REM sleep. ${ }^{25,26}$

In neonatal animals, this spontaneous firing is very low, which partly explains the dominance of REM sleep in human premature and term infants. Developing neonatal rodents show a graduated increase in arousal and wake-bout duration. ${ }^{27-29}$ Increased locus coeruleus neuronal firing can promote wakefulness by pressing noradrenergic inhibition in the ventrolateral preoptic area of the hypothalamus. ${ }^{25,30}$ Therefore, with the increase in age, the increased firing rate of the locus coeruleus and the noradrenergic innervation of brain may be the reasons for the increase in wakefulness with maturation. ${ }^{31,32}$ This may explain why the percentage and duration of wakefulness increased at P12.

Sleep-wake cycles in human infants have been related to cognitive performance and sensorimotor outcomes. ${ }^{33-35}$ The development and length of sleep-wake cycles are also influenced by neurologic injuries in human preterm and term infants. ${ }^{6,36}$ In animal models, it has been demonstrated that although the locus coeruleus neurons have low firing frequency, these neurons can fire strongly following nociceptive stimuli. ${ }^{37-39}$ Reciprocal stimulation occurs between 
corticotropin-releasing hormone $(\mathrm{CRH})$ release and locus coeruleus neuronal firing and between $\mathrm{CRH}$ release and the orexinergic neuronal activity, which increases locus coeruleus firing. ${ }^{40-43}$ This is consistent with results that wakefulness can be triggered by nociception in human infants. ${ }^{17,44}$ In our study, the percentage of wake time in the pups in the HI group was significantly increased after HI induction. This indicated that HI brain injury may prolong wakefulness by influencing these positive feedback circuits.

Overall, our findings of the HIE model in neonatal rats correlate with most features of the EEG and sleep-wake patterns of human preterm infants of previous studies. However, this study had some limitations. First, the recording length was inadequate to acquire more sleep-wake cycles, as neonatal rat pups must live with their mothers for breastfeeding support and cannot be separated from the dam for a long time. Although it has been studied that shortperiod newborn neonatal sleep-wake recording can represent long-term recordings, ${ }^{45}$ future studies should aim to develop smaller radio electrodes and record EEG data from multiple brain regions for 24 hours to ideally obtained serial data and more detailed information. A second limitation is that recordings were only performed during the light phase, which may result in secondary to circadian rhythms bias. However, previous studies have indicated that rats mostly do not manifest consolidated circadian rhythms before P17. ${ }^{46,47}$ Studies of sleep-wake cycle can support a relative accurate classification of neonatal encephalopathy that does not meet standards for HIE. ${ }^{6}$ Despite this limitation, our technique for obtaining EEG and EMG data of neonatal rats allowed repetitive, longitudinal sleep-wake assessments during the crucial period of brain development and the early period effects of HIE disease.

\section{Abbreviations}

EEG, electroencephalogram; EMG, electromyogram; HI, hypoxia-ischemia; HIE, hypoxic-ischemic encephalopathy; NREM, non-rapid eye movement; REM, rapid eye movement.

\section{Acknowledgments}

We would like to thank the In Vivo Electrophysiology Laboratory of Core Facility Center (Capital Medical University, Beijing, China) for help with electrode fabrication and data analysis. We would like to thank Editage for English language editing.

\section{Funding}

This work was supported by a grant from the National Natural Science Foundation of China (No. 81771622 and No. 82171693 to Hong Cui) and the Natural Science Foundation of Beijing Municipality (No. 7202035 to Lijun Yang).

\section{Disclosure}

The authors declare that they have no known competing financial interests or personal relationships that could have appeared to influence the work reported in this paper.

\section{References}

1. Ophelders D, Gussenhoven R, Klein L, et al. Preterm brain injury, antenatal triggers, and therapeutics: timing is key. Cells. $2020 ; 9(8): 746$. doi:10.3390/cells9081871

2. Galinsky R, Lear CA, Dean JM, et al. Complex interactions between hypoxia-ischemia and inflammation in preterm brain injury. Dev Med Child Neurol. 2018;60(2):126-133. doi:10.1111/dmcn.13629

3. Manuck TA, Rice MM, Bailit JL, et al. Preterm neonatal morbidity and mortality by gestational age: a contemporary cohort. Am J Obstet Gynecol. 2016;215(1):103e1-103 e14. doi:10.1016/j.ajog.2016.01.004

4. Graham EM, Ruis KA, Hartman AL, Northington FJ, Fox HE. A systematic review of the role of intrapartum hypoxia-ischemia in the causation of neonatal encephalopathy. Am J Obstet Gynecol. 2008;199(6):587-595. doi:10.1016/j.ajog.2008.06.094

5. Douglas-Escobar M, Weiss MD. Hypoxic-ischemic encephalopathy: a review for the clinician. JAMA Pediatr. 2015;169(4):397-403. doi:10.1001/ jamapediatrics.2014.3269

6. Scher MS, Steppe DA, Beggarly ME, Salerno DG, Banks DL. Neonatal EEG-sleep disruption mimicking hypoxic-ischemic encephalopathy after intrapartum asphyxia. Sleep Med. 2002;3(5):411-415. doi:10.1016/s1389-9457(02)00071-0

7. Pace-Schott EF, Hobson JA. The neurobiology of sleep: genetics, cellular physiology and subcortical networks. Nat Rev Neurosci. 2002;3(8):591605. doi:10.1038/nrn895

8. Wolfe K, Ralls FM. Rapid eye movement sleep and neuronal development. Curr Opin Pulm Med. 2019;25(6):555-560. doi:10.1097/ MCP.0000000000000622 
9. Scammell TE, Arrigoni E, Lipton JO. Neural Circuitry of Wakefulness and Sleep. Neuron. 2017;93(4):747-765. doi:10.1016/j.neuron.2017.01.014

10. Skranes JH, Lohaugen G, Schumacher EM, et al. Amplitude-Integrated Electroencephalography Improves the Identification of Infants with Encephalopathy for Therapeutic Hypothermia and Predicts Neurodevelopmental Outcomes at 2 Years of Age. J Pediatr. 2017;187:34-42. doi:10.1016/j.jpeds.2017.04.041

11. Takenouchi T, Rubens EO, Yap VL, Ross G, Engel M, Perlman JM. Delayed onset of sleep-wake cycling with favorable outcome in hypothermictreated neonates with encephalopathy. J Pediatr. 2011;159(2):232-237. doi:10.1016/j.jpeds.2011.01.006

12. Shany E, Goldstein E, Khvatskin S, et al. Predictive value of amplitude-integrated electroencephalography pattern and voltage in asphyxiated term infants. Pediatr Neurol. 2006;35(5):335-342. doi:10.1016/j.pediatrneurol.2006.06.007

13. Aswathy BS, Kumar VM, Gulia KK. The effects of rapid eye movement sleep deprivation during late pregnancy on newborns' sleep. $J$ Sleep Res. 2018;27(2):197-205. doi:10.1111/jsr.12564

14. Rensing N, Moy B, Friedman JL, Galindo R, Wong M. Longitudinal analysis of developmental changes in electroencephalography patterns and sleep-wake states of the neonatal mouse. PLoS One. 2018;13(11):e0207031. doi:10.1371/journal.pone.0207031

15. Sizonenko SV, Sirimanne E, Mayall Y, Gluckman PD, Inder T, Williams C. Selective cortical alteration after hypoxic-ischemic injury in the very immature rat brain. Pediatr Res. 2003;54(2):263-269. doi:10.1203/01.PDR.0000072517.01207.87

16. Stadlin A, James A, Fiscus R, Wong YF, Rogers M, Haines C. Development of a postnatal 3-day-old rat model of mild hypoxic-ischemic brain injury. Brain Res. 2003;993(1-2):101-110. doi:10.1016/j.brainres.2003.08.058

17. Georgoulas A, Jones L, Laudiano-Dray MP, Meek J, Fabrizi L, Whitehead K. Sleep-wake regulation in preterm and term infants. Sleep. 2021;44 (1):98. doi:10.1093/sleep/zsaa148

18. Yang G, Gan WB. Sleep contributes to dendritic spine formation and elimination in the developing mouse somatosensory cortex. Dev Neurobiol. 2012;72(11):1391-1398. doi:10.1002/dneu.20996

19. Dooley JC, Blumberg MS. Developmental 'awakening' of primary motor cortex to the sensory consequences of movement. Elife. $2018 ; 7: 537$. doi:10.7554/eLife.41841

20. Mukherjee D, Yonk AJ, Sokoloff G, Blumberg MS. Wakefulness suppresses retinal wave-related neural activity in visual cortex. J Neurophysiol. 2017;118(2):1190-1197. doi:10.1152/jn.00264.2017

21. An S, Kilb W, Luhmann HJ. Sensory-evoked and spontaneous gamma and spindle bursts in neonatal rat motor cortex. J Neurosci. 2014;34 (33):10870-10883. doi:10.1523/JNEUROSCI.4539-13.2014

22. Frank MG. Sleep and plasticity in the visual cortex: more than meets the eye. Curr Opin Neurobiol. 2017;44:8-12. doi:10.1016/j.conb.2017.01.001

23. Milh M, Kaminska A, Huon C, Lapillonne A, Ben-Ari Y, Khazipov R. Rapid cortical oscillations and early motor activity in premature human neonate. Cereb Cortex. 2007;17(7):1582-1594. doi:10.1093/cercor/bhl069

24. Fifer WP, Byrd DL, Kaku M, et al. Newborn infants learn during sleep. Proc Natl Acad Sci U S A. 2010;107(22):10320-10323. doi:10.1073/ pnas. 1005061107

25. Saper CB, Fuller PM, Pedersen NP, Lu J, Scammell TE. Sleep state switching. Neuron. 2010;68(6):1023-1042. doi:10.1016/j.neuron.2010.11.032

26. Diniz Behn CG, Booth V. Simulating microinjection experiments in a novel model of the rat sleep-wake regulatory network. $J$ Neurophysiol. 2010;103(4):1937-1953. doi:10.1152/jn.00795.2009

27. Blumberg MS, Coleman CM, Johnson ED, Shaw C. Developmental divergence of sleep-wake patterns in orexin knockout and wild-type mice. Eur J Neurosci. 2007;25(2):512-518. doi:10.1111/j.1460-9568.2006.05292.x

28. Daszuta A, Gambarelli F. Early postnatal development of EEG and sleep-waking cycle in two inbred mouse strains. Brain Res. 1985;354(1):39-47. doi:10.1016/0165-3806(85)90066-5

29. Blumberg MS, Seelke AM, Lowen SB, Karlsson KA. Dynamics of sleep-wake cyclicity in developing rats. Proc Natl Acad Sci U S A. 2005;102 (41):14860-14864. doi:10.1073/pnas.0506340102

30. Szymusiak R, Alam N, Steininger TL, McGinty D. Sleep-waking discharge patterns of ventrolateral preoptic/anterior hypothalamic neurons in rats. Brain Res. 1998;803(1-2):178-188. doi:10.1016/s0006-8993(98)00631-3

31. Nakamura S, Sakaguchi T. Development and plasticity of the locus coeruleus: a review of recent physiological and pharmacological experimentation. Prog Neurobiol. 1990;34(6):505-526. doi:10.1016/0301-0082(90)90018-c

32. Levitt P. Structural and functional maturation of the developing primate brain. J Pediatr. 2003;143(4 Suppl):S35-45. doi:10.1067/s0022-3476(03) 00400-1

33. Wikstrom S, Pupp IH, Rosen I, et al. Early single-channel aEEG/EEG predicts outcome in very preterm infants. Acta Paediatr. 2012;101(7):719726. doi:10.1111/j.1651-2227.2012.02677.x

34. Hellstrom-Westas L, Rosen I, Svenningsen NW. Cerebral function monitoring during the first week of life in extremely small low birthweight (ESLBW) infants. Neuropediatrics. 1991;22(1):27-32. doi:10.1055/s-2008-1071411

35. Shellhaas RA, Burns JW, Hassan F, Carlson MD, Barks JDE, Chervin RD. Neonatal Sleep-Wake Analyses Predict 18-month Neurodevelopmental Outcomes. Sleep. 2017;40:11. doi:10.1093/sleep/zsx144

36. Olischar M, Klebermass K, Waldhoer T, Pollak A, Weninger M. Background patterns and sleep-wake cycles on amplitude-integrated electroencephalography in preterms younger than 30 weeks gestational age with peri-/intraventricular haemorrhage. Acta Paediatr. 2007;96(12):17431750. doi:10.1111/j.1651-2227.2007.00462.x

37. Foote SL, Aston-Jones G, Bloom FE. Impulse activity of locus coeruleus neurons in awake rats and monkeys is a function of sensory stimulation and arousal. Proc Natl Acad Sci U S A. 1980;77(5):3033-3037. doi:10.1073/pnas.77.5.3033

38. Fulcher BD, Phillips AJ, Robinson PA. Modeling the impact of impulsive stimuli on sleep-wake dynamics. Phys Rev E Stat Nonlin Soft Matter Phys. 2008;78(5 Pt 1):051920. doi:10.1103/PhysRevE.78.051920

39. Takahashi K, Kayama Y, Lin JS, Sakai K. Locus coeruleus neuronal activity during the sleep-waking cycle in mice. Neuroscience. 2010;169 (3):1115-1126. doi:10.1016/j.neuroscience.2010.06.009

40. Dunn AJ, Swiergiel AH, Palamarchouk V. Brain circuits involved in corticotropin-releasing factor-norepinephrine interactions during stress. Ann $N$ Y Acad Sci. 2004;1018:25-34. doi:10.1196/annals.1296.003

41. Yamanaka A, Beuckmann CT, Willie JT, et al. Hypothalamic orexin neurons regulate arousal according to energy balance in mice. Neuron. 2003;38 (5):701-713. doi:10.1016/s0896-6273(03)00331-3 
42. Huppertz-Kessler CJ, Poeschl J, Hertel R, Unsicker K, Schenkel J. Effects of a new postnatal stress model on monoaminergic neurotransmitters in rat brains. Brain Dev. 2012;34(4):274-279. doi:10.1016/j.braindev.2011.07.008

43. Cano G, Mochizuki T, Saper CB. Neural circuitry of stress-induced insomnia in rats. J Neurosci. 2008;28(40):10167-10184. doi:10.1523/ JNEUROSCI.1809-08.2008

44. Anders TF, Roffwarg HP. The effects of selective interruption and deprivation of sleep in the human newborn. Dev Psychobiol. 1973;6(1):77-89. doi: $10.1002 /$ dev. 420060110

45. Stern E, Parmelee AH, Harris MA. Sleep state periodicity in prematures and young infants. Dev Psychobiol. 1973;6(4):357-365. doi:10.1002/ dev.420060409

46. Frank MG, Ruby NF, Heller HC, Franken P. Development of Circadian Sleep Regulation in the Rat: a Longitudinal Study Under Constant Conditions. Sleep. 2017;40(3):245. doi:10.1093/sleep/zsw077

47. Frank MG, Heller HC. Development of diurnal organization of EEG slow-wave activity and slow-wave sleep in the rat. Am J Physiol. 1997;273(2 Pt 2): R472-8. doi:10.1152/ajpregu.1997.273.2.R472

\section{Publish your work in this journal}

Nature and Science of Sleep is an international, peer-reviewed, open access journal covering all aspects of sleep science and sleep medicine, including the neurophysiology and functions of sleep, the genetics of sleep, sleep and society, biological rhythms, dreaming, sleep disorders and therapy, and strategies to optimize healthy sleep. The manuscript management system is completely online and includes a very quick and fair peer-review system, which is all easy to use. Visit http://www.dovepress.com/testimonials.php to read real quotes from published authors.

Submit your manuscript here: https://www.dovepress.com/nature-and-science-of-sleep-journal 